\title{
De I'immunologie fondamentale au développement de la vaccinologie
}

$>$ Ces dix dernières années, la recherche en vaccinologie s'est développée au niveau mondial, et s'il faut encore promouvoir les vaccins empiriques mais efficaces, il paraît nécessaire de mettre au point des vaccins de nouvelle génération contre des infections comme le VIH/Sida. Jean-Gérard Guillet fut un pionnier en la matière car, en partant de ses premiers résultats sur la présentation des peptides antigéniques aux lymphocytes $T$, il a orienté ses recherches sur les mécanismes d'induction de la réponse cellulaire T et sur la sélection des peptides antigéniques pour élaborer de nouveaux vaccins à base de lipopeptides. Leur potentiel immunogène a été testé sur des modèles animaux (souris, singe) et les premiers essais de vaccination chez l'homme ont été promus par l'ANRS. L'étude des réponses immunitaires induites chez les vaccinés a pu se développer grâce à la création d'une plate-forme d'immunomonitorage et du Centre d'Investigation Clinique Cochin/Pasteur spécialisé en vaccinologie. $<$

Cet article est dédié à la mémoire de Jean-Gérard Guillet
Jeannine Choppin

In memoriam Jean-Gérard Guillet

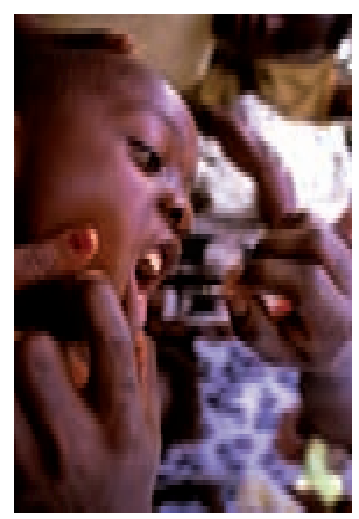

Département d'Immunologie, Institut Cochin, Inserm U567, CNRS (UMR 8104), Université Paris Descartes, Bâtiment Hardy A, 27, rue du Faubourg Saint-Jacques, 75014 Paris, France. choppin@cochin.inserm.fr

les anticorps induits étaient inefficaces vis-à-vis des souches de virus circulantes. L'objectif des programmes de recherche suivants a été d'induire des réponses cellulaires faisant intervenir les lymphocytes $T$ auxiliaires CD4 et cytotoxiques CD8. Un vaccin basé sur les réponses cellulaires est alors plutôt conçu comme un vaccin de première génération d'efficacité partielle qui diminuerait la charge virale après la primoinfection et permettrait d'obtenir une longue période d'évolution asymptomatique de la maladie. Les effets bénéfiques attendus sont l'épargne pendant plusieurs années de médicaments anti-viraux très coûteux et entraînant des troubles secondaires multiples, et la diminution de la charge virale moyenne au niveau des populations, qui s'accompagnerait d'une réduction de la transmission. La mise en place d'une telle stratégie est bien illustrée par l'ensemble des travaux promus avec un grand dynamisme par Jean-Gérard Guillet qui nous a quittés le 7 mai 2007.

\section{Travaux sur la présentation des peptides antigéniques aux lymphocytes T}

Des travaux publiés dans les années 1986-1989 ont fortement contribué à élucider les bases moléculaires du phénomène de restriction de la présentation des antigènes par le complexe majeur d'histocompatibilité (CMH) et de la reconnaissance, par les lymphocytes T, 
de tout antigène étranger sous forme de peptides présentés par les molécules du CMH ou antigènes de restriction du soi. Une question cruciale était alors posée : comment des peptides très divers, issus d'un nombre incalculable de protéines étrangères, pouvaient-ils former des complexes uniques avec un nombre limité de molécules du CMH ? Les travaux de Jean-Gérard Guillet, alors en poste au Massachusetts Institute of Technology, Cambridge (États-Unis), ont éclairci les mécanismes de la reconnaissance par les lymphocytes $T$ CD4 des peptides présentés sur des molécules de classe II du CMH. Aussi bien les peptides analogues au peptide initial utilisé pour la stimulation des lymphocytes $T$, que des peptides dérivés d'autres antigènes, pouvaient inhiber de manière compétitive l'activité des lymphocytes $T$ [4]. Ces constatations ont conduit à proposer un modèle: dans la cellule présentatrice, les peptides issus de tous les antigènes dégradés se fixent sur un même site de la molécule du $\mathrm{CMH}$, et la présence de ce site polymorphique, assurant la liaison de peptides très variés, peut expliquer la sélection du soi dans le thymus et la restriction par le CMH [5]. Peu après, I'association physique entre divers peptides immunogènes et les molécules de classe I du CMH humain, le système HLA, a été démontrée dans I'U152 Inserm de Jean-Paul Lévy à I'hôpital Cochin $[6,7]$. Les travaux ont été poursuivis dans I'U445 Inserm dirigée par Jean-Gérard Guillet et un test permettant de détecter les interactions peptide/HLA de classe I a été mis au point [8]. Des peptides de 8 à 10 résidus, majoritairement de 9 résidus, se fixent aux molécules HLA de classe I et diverses séries de peptides interagissent sélectivement avec les différents types de molécules HLA. Cette interaction est conditionnée par la charge des peptides et la présence de résidus hydrophobes; toutefois, des différences quantitatives (concentration active très variable selon le peptide considéré) peuvent faciliter la formation de certains types de complexes HLA/peptide. II a aussi été montré que des modifications de la structure primaire des épitopes par des acides aminés naturels, ou l'emploi d'analogues, peuvent avoir un impact sur l'interaction peptide/HLA et les fonctions des cellules T [9]. Enfin, un autre point particulièrement important a été l'observation que les peptides reconnus par les lymphocytes $T$ peuvent être regroupés dans certaines régions des antigènes dites régions poly-épitopiques [10]. Toutes ces données ont constitué la base de la stratégie vaccinale qui a été développée dans le laboratoire de Jean-Gérard Guillet.

\section{Caractéristiques des réponses T anti-VIH observées chez les sujets infectés}

Le caractère immunogène des fragments d'antigènes issus du VIH a d'abord été montré par la mise en évidence de nombreuses réponses T CD8 cytotoxiques chez les sujets infectés. Ainsi la région centrale de la protéine Nef du VIH a été décrite comme une cible préférentielle de ces lymphocytes T et de multiples épitopes ont été détectés [11]. L'étude systématique des réponses lymphocytaires d'une série de patients a permis d'analyser la variabilité virale des épitopes de Nef et a révélé que certaines variations apparaissaient en parallèle avec une forte réduction de la reconnaissance des lymphocytes $T$ et semblaient responsables de l'échappement du virus à la réponse immunitaire [12]. Par ailleurs, à partir d'une cohorte de sujets infectés chroniquement, l'équipe de Jean-Gérard Guillet a montré que de fortes réponses des lymphocytes T CD8 dirigées simultanément contre plusieurs peptides du VIH ne pouvaient pas contrôler la virémie [13]. II semblait alors important de connaître avec plus de précision les réponses primaires anti-VIH et de déterminer les caractéristiques de la réponse T et la nature des épitopes ciblés lors de l'observation d'une protection contre l'infection, comme cela apparaissait chez quelques sujets résistants.

\section{De la sélection des épitopes à l'élaboration des lipopeptides vaccinaux}

Dans le contexte d'une stratégie vaccinale à base de fragments antigéniques immunogènes il était nécessaire d'utiliser des parties de protéines de longueur suffisante (de 20 à 40 résidus) pour contenir une série d'épitopes aptes à être présentés par plusieurs types de molécules HLA de classe I et II. A priori, les molécules HLA les plus intéressantes étaient les plus fréquentes dans les populations car il fallait chercher à induire des réponses immunes chez la majorité des sujets vaccinés. Un autre problème à résoudre était d'éviter le risque d'échappement lié au caractère particulièrement variable de virus comme le $\mathrm{VIH}$, et les résultats des travaux réalisés dans le laboratoire de Jean-Gérard Guillet ont permis de sélectionner les régions les plus conservées des protéines comme certaines régions de Gag ou la partie centrale de Nef. L'inclusion de peptides dans les préparations vaccinales était une approche alternative à l'utilisation de vecteurs recombinants, avec comme atouts majeurs la spécificité de la réponse et le faible niveau de risque. Pour conférer une immunogénicité suffisante, l'emploi d'adjuvants ainsi que le couplage d'une extrémité de chaque peptide d'intérêt à une molécule lipidique simple comprenant un groupe palmitoyl ont été réalisés, ce qui a abouti à la fabrication de lipopeptides. La mise en application du concept des lipopeptides a pu être effective grâce à l'instauration de collaborations avec des industriels, dont des laboratoires pharmaceutiques, et aussi avec des unités de recherche spécialisées dans la chimie des biomolécules et peptides (Institut Pasteur/CNRS 1309, Lille; J.P. Briand/S. Muller, CNRS UPR 9021, Strasbourg, France).

Les premiers essais de vaccination avec des fragments d'antigènes viraux ont été réalisés chez la 
souris. Le potentiel immunogène des lipopeptides a d'abord été démontré par l'induction de réponses cytolytiques primaires contre un épitope de l'enveloppe du VIH [14]. L'effet de l'addition de divers lipides à des fragments d'enveloppe sur la réponse T CD8 a ensuite alors été étudié en détail [15]. Des lipopeptides contenant des séquences de 16 à 34 résidus regroupant plusieurs épitopes ont induit des réponses $T$ multiples cytolytiques et proliférantes, et ont produit des anticorps, révélant la possibilité d'obtenir une réponse immune totale. Les essais réalisés chez le Macaque avec un mélange de 7 lipopeptides issus des protéines Nef et Gag du virus du singe ont montré l'obtention de réponses cellulaires T soutenues et la production d'anticorps [16]. Les réponses ont ensuite été optimisées par l'addition d'un épitope auxiliaire (helper) provenant de la toxine tétanique [17]. D'autres modèles de vaccination ont aussi été testés, et par exemple, les lipopeptides utilisés pour une vaccination contre le mélanome ont induit de fortes réponses T cytotoxiques chez la souris transgénique pour le HLA-A2 [18].

Dans les années 1995-2000, beaucoup de travaux ont porté sur l'apprêtement des antigènes. On a montré notamment que les conditions modulant la libération d'épitopes d'intérêt et l'effet de molécules accessoires sur l'assemblage $\mathrm{CMH}$ /peptide agissaient sur le profil de restriction HLA au niveau des cellules présentatrices d'antigènes et sur la nature des lymphocytes T engendrés. Pour comprendre les mécanismes d'induction des réponses T par les lipopeptides, la dégradation in vitro des fragments d'antigènes du VIH inclus dans les préparations vaccinales par des systèmes enzymatiques cellulaires de référence a été réalisée et la possibilité de produire des épitopes d'intérêt a été mise en évidence $[19,20]$. Dans la même optique, le devenir des lipopeptides au niveau de la cellule dendritique responsable de la stimulation primaire des lymphocytes T a été étudié [21]. La partie lipidique du lipopeptide lui permet d'entrer d'une manière non spécifique dans n'importe quelle cellule dendritique. Après leur endocytose, les différents épitopes peuvent être libérés puis présentés par les molécules du CMH de classe I. Par microscopie confocale, on a observé que les voies cytoplasmiques et endosomiques étaient utilisées pour aboutir à la présentation des épitopes aux lymphocytes T.

Enfin, pour augmenter l'immunogénicité des préparations, des modifications des lipopeptides, comme l'introduction de nouveaux fragments d'antigènes ou l'administration conjointe d'adénovirus, ont été réalisées. Le rôle adjuvant de l'adénovirus $A d 5$, administré avec des lipopeptides, a été démontré chez la souris qui a développé des réponses T CD4 et CD8. En testant les divers composants de la capside, l'hexon s'est révélé porteur d'un effet auxiliaire important [22]. L'adénovirus a aussi induit une maturation complète de cellules dendritiques murines. Des tests faits à l'aide de protéines recombinantes de capside ou de certaines régions de ces protéines, ont permis d'établir que cet effet était causé par la fibre [23]. Par la suite, dans un travail collaboratif avec B. Frisch (CNRS UMR 7514, Strasbourg, France), d'autres adjuvants similaires aux lipopeptides bactériens ont été construits et ont donné des effets stimulants intéressants, qui sont encore en cours d'étude.

\section{Les premiers essais de vaccination réalisés chez l'homme avec l'Agence Nationale de la Recherche sur le Sida et les hépatites (ANRS)}

Le travail réalisé sur les vaccins par le groupe de JeanGérard Guillet a essentiellement porté sur des lipopeptides. Par exemple, dans l'essai de phase I, ANRS VAC 04, 28 volontaires sains ont reçu 3 injections de 6 lipopeptides dérivés des protéines Nef, Gag et Env du VIH et la tolérance a été bonne. On a constaté des réponses anticorps chez $90 \%$ des sujets, et chez $80 \%$ des sujets des réponses T CD4 ayant de multiples spécificités, ainsi que de nombreuses réponses T CD8 anti-Gag et anti-Nef de longue durée [24, 25]. Dans les protocoles vaccinaux de l'ANRS engagés avec les lipopeptides, d'autres formulations ont été testées, incluant la présence de l'épitope auxiliaire de la toxine tétanique, ou un vecteur ALVAC, dérivé du Canari Pox. Des injections de rappel et différentes doses ou voies d'injection ont aussi été programmées [26, 27]. En vaccination thérapeutique, chez des sujets infectés par le VIH, les lipopeptides ont induit de nouvelles réponses anti-VIH relayées par les cellules T CD4 et CD8 [28]. L'impact positif de la vaccination par l'ALVAC et les lipopeptides, suivie par des injections d'IL-2 chez des sujets infectés après l'arrêt des traitements antiviraux a été d'induire des réponses T CD4 et CD8 anti-VIH associées à un contrôle de la virémie [29]. Pour traiter les échantillons et interpréter les résultats de ces essais, et aussi expliquer les mécanismes de ces réponses sur un plan fondamental, Jean-Gérard Guillet a créé une plate-forme d'évaluation de la réponse immunitaire. Dans le même temps, en collaboration étroite avec Jean-Gérard Guillet, s'est développé, à I'hôpital Cochin, le Centre d'Investigation Clinique de Vaccinologie Cochin/Pasteur, seul centre de recherche clinique français spécialisé en vaccinologie.

\section{Bilan actuel sur les projets de vaccination anti-VIH}

Les premiers résultats des essais vaccinaux ont montré la possibilité d'obtenir des réponses immunes cellulaires $T$ contre des fragments de plusieurs protéines $\mathrm{du}$ VIH. Les mises au point des vaccins sont poursuivies pour tenter d'amplifier les réponses et surtout de les maintenir à long terme. Les protocoles sont affinés par la recherche de préparations vaccinales comportant les associations les plus performantes, des voies et des fréquences optimales d'administration. La capacité de protection de ces nouveaux vaccins ne peut pas encore être démontrée dans l'infection par le $\mathrm{VIH}$ et il faut tenir compte de l'extrême variabilité des 
souches de virus circulantes dans le monde. II faut encore affiner la sélection des fragments protéiques à insérer dans les vaccins pour obtenir des réponses croisées, c'est-à-dire ciblant la majorité des clades de virus. Cet aspect fait l'objet d'un vaste projet de recherche international avec le National Institute of Health (Bethesda, ÉtatsUnis), auquel Jean-Gérard Guillet était associé. Une partie du programme porte sur les mécanismes des réponses immunes $T$ induites par la vaccination, la nature des lymphocytes $T$ induits, les épitopes ciblés, la dominance des réponses et les molécules HLA concernées, en comparaison avec les réponses observées chez des sujets infectés. L'équipe de Jean-Gérard Guillet s'était fait une spécialité de cette approche [30]. Enfin, pour établir des corrélats généraux et fiables de protection antivirale, les réponses immunes cellulaires T efficaces contre d'autres types de virus doivent être analysées; la nature des lymphocytes $T$ aptes à induire une protection (marqueurs et fonction sécrétoire) doit être définie. Les réponses cellulaires présentes chez les individus qui contrôlent bien et à long terme l'infection par le VIH peuvent aussi donner des informations importantes, notamment sur les régions des protéines virales à cibler de préférence et à introduire dans les futurs vaccins.

Au total, depuis les aspects les plus fondamentaux des conséquences fonctionnelles de l'interaction des épitopes peptidiques avec les molécules du CMH jusqu'au transfert de ces données en recherche vaccinale, l'œuvre scientifique de Jean-Gérard Guillet témoigne d'une impressionnante cohérence. Cette dernière est même renforcée par l'implication de ce chercheur dans les essais cliniques, la création d'une plate-forme de suivi des réponses immunitaires aux candidats vaccins et d'un Centre d'Investigation Clinique dédié. $\diamond$

\section{SUMMARY}

From fundamental immunology to development of vaccinology In the last ten years research in vaccinology has been developed in the world to conceive new vaccine approaches against infections like HIV/AIDS. Jean-Gérard Guillet is a pioneer in the development of new vaccine strategies. From the first results he obtained in the late 80 's on the presentation of antigenic peptides to T cells, he axed his work on the study of induction mechanisms of $T$ cell mediated immune responses. The selection of antigenic peptides and the search to enhance antigen immunogenicity led him to elaborate lipopeptides as new vaccine formulae. The efficacy of these preparations was tested in animal models (mouse, macaque) and, thereafter, in humans with clinical trials promoted by the French National Agency for AIDS and viral hepatitis (ANRS). The study of T-cell induced responses in vaccinated volunteers was implemented following the creation of two facilities, an immuno-monitoring platform and the Clinical Investigation Centre Cochin-Pasteur, a structure specialized in vaccinology. $\diamond$

\section{REMERCIEMENTS}

Je tiens à remercier le Pr Axel Kahn pour ses conseils lors de la rédaction de cet article.

\section{LA RECHERCHE EN VACCINOLOGIE}

L'approche globale du concept de vaccinologie fondamentale et clinique en France est encore peu organisée, comme l'a souligné dernièrement $P$. Sansonetti dans un éditorial de Médecine/Sciences [1]. La recherche en vaccinologie demande la conjugaison de plusieurs thématiques portant sur l'immunologie fondamentale, les maladies infectieuses, l'épidémiologie et la création de nouveaux vaccins qui nécessite des partenariats industriels et des essais cliniques conséquents. II est nécessaire de développer des centres d'investigation clinique (CIC), spécialisés en vaccinologie comme celui de Cochin/Pasteur qui résulte d'un partenariat établi entre I'Inserm, I'Institut Pasteur, I'AP-Hôpitaux de Paris et I'Université Paris Descartes. Les multiples travaux de Jean-Gérard Guillet l'ont amené à concevoir les lipopeptides comme des vaccins et son énergie a été canalisée sur l'établissement de réseaux pour mettre en commun les compétences de divers experts (chimistes, pharmacologues...), pour entretenir des collaborations internationales, seul moyen de lutte efficace contre les fléaux mondiaux et pour assurer le maintien d'un dialogue permanent avec les cliniciens. $\mathrm{Sa}$ recherche fut exemplaire et, dans les protocoles vaccinaux contre le VIH de l'ANRS, des résultats ont été obtenus sur sa plate-forme d'immunomonitorage, qui est étroitement liée au CIC et à son équipe de recherche. La compréhension et l'exploitation de ces résultats nécessitent bien un retour constant vers l'immunologie fondamentale.

\section{RÉFÉRENCES}

1. Sansonetti P. Plaidoyer pro domo vaccino. Med Sci (Paris) 2007 ; $23: 342-4$

2. Kaufmann SHE, McMichael AJ. Annulling a dangerous liaison : vaccination strategies against AIDS and tuberculosis. Nat Med 2005; 11 : S33-44.

3. Leclerc C. L'apport des nouvelles technologies en vaccinologie. Med Sci (Paris) $2007 ; 23: 386-90$.

4. Guillet JG, Lai MZ, Briner TJ, et al. Interaction of peptide antigens and class II major histocompatibility complex antigen. Nature 1986; $324: 260-2$.

5. Guillet JG, Lai MZ, Briner TJ, et al. Immunological self, nonself discrimination. Science $1987 ; 235: 865-70$.

6. Bouillot M, Choppin J, Cornille F, et al. Physical association between MHC molecules and immunogenic peptides. Nature 1989 ; 339 : 473-5.

7. Choppin J, Martinon F, Gomard $\varepsilon$, et al. Analysis of physical interactions between peptides and HLA molecules and application to the detection of human immunodeficiency virus 1 antigenic peptides. J Exp Med 1990 ; $172: 889-99$.

8. Connan F, Hlavac F, Hoebeke J, et al. A simple assay for detection of peptides promoting the assembly of HLA class I molecules. Eur I Immunol $1994 ; 24: 777-80$.

9. Ostankovitch M, Guichard G, Connan F, et al. A partially modified retroinverso pseudopeptide modulates the cytokine profile of CTL specific for an influenza virus epitope. J Immunol 1998; $161: 200-8$.

10. Choppin J, Cohen W, Bianco A, et al. Characteristics of HIV-1 Nef regions containing multiple CD8 ${ }^{+} \mathrm{T}$ cell epitopes : wealth of HLA-binding motifs and sensitivity to proteasome degradation. J Immunol 2001; $166: 6164-9$ 
11. Culmann-Penciolelli B, Lamhamedi-Cherradi S, Couillin I, et al. Identification of multirestricted immunodominant regions recognized by cytolytic T lymphocytes in the human immunodeficiency virus type 1 nef protein. J Virol $1994 ; 68: 7336-43$.

12. Couillin I, Culmann-Penciolelli B, Gomard E, et al. Impaired cytotoxic T lymphocyte recognition due to genetic variations in the main immunogenic region of the human immunodeficiency virus 1 Nef protein. J Exp Med 1994; 180 : 1129-34.

13. Dalod M, Dupuis $M$, Deschemin JC, et al. Broad, intense anti-human immunodeficiency virus (HIV) ex vivo $\mathrm{CD}^{+}$responses in HIV type l-infected patients : comparison with anti-EpsteinBarr virus responses and changes during antiretroviral therapy. J Virol 1999; 73: 7108-16.

14. Martinon F, Gras-Masse H, Boutillon C, et al. Immunization of mice with lipopeptides bypasses the prerequisite for adjuvant. Immune response of BALB/c mice to human immunodeficiency virus envelope glycoprotein. J Immunol $1992 ; 149$ : 3416-22

15. Deprez B, Sauzet JP, Boutillon C, et al. Comparative efficiency of simple lipopeptide constructs for in vivo induction of virus-specific CTL. Vaccine $1996 ; 14: 375-82$.

16. Bourgault I, Chirat F, Tartar A, et al. Simian immunodeficiency virus as a model for vaccination against HIV. Induction in rhesus macaques of GAG- or NEF-specific cytotoxic T lymphocytes by lipopeptides. J Immunol $1994 ; 152: 2530-7$.

17. Mortara L, Gras Masse H, Rommens C, et al. Type $1 \mathrm{CD}^{+} \mathrm{T}$-cell help is required for induction of anti-peptide multispecific cytotoxic $T$ lymphocytes by a lipopeptidic vaccine in rhesus macaques. J Virol $1999 ; 73$ : 4447-51.

18. Le Gal FA, Prevost-Blondel A, Lengagne $R$, et al. Lipopeptide-based melanoma cancer vaccine induced a strong MART-27-35-cytotoxic T lymphocyte response in a preclinal study. Int Cancer $2002 ; 98: 221-7$.

19. Choppin J, Cohen W, Bianco A, et al. Characteristics of HIV-1 Nef regions containing multiple CD8 ${ }^{+}$T cell epitopes : wealth of HLA-binding motifs and sensitivity to proteasome degradation. J Immunol $2001 ; 166: 6164-9$.

20. Cohen W, Bianco A, Connan F, et al. Study of antigen-processing steps reveals preferences explaining differential biological outcomes of two HLA-A2-restricted immunodominant epitopes from human immunodeficiency virus type 1.J Virol $2002 ; 76$ : 10219-25.

21. Andrieu M, Desoutter JF, Loing $\varepsilon$, et al. Two human immunodeficiency virus vaccinal lipopeptides follow different cross-presentation pathways in human dendritic cells. J Viro $2003 ; 77: 1564-70$
22. Molinier-Frenkel V, Lengagne R, Gaden F, et al. Adenovirus hexon protein is a potent adjuvant for activation of a cellular immune response. J Virol $2002 ; 76: 127-35$

23. Molinier-Frenkel V, Prevost-Blondel A, Hong SS, et al. The maturation of murine dendritic cells induced by human adenovirus is mediated by the fiber knob domain. J Biol Chem 2003; 278 : 37175-82.

24. Gahery-Segard H, Pialoux G, Charmeteau B, et al. Multiepitopic B- and $T$-cell responses induced in humans by a human immunodeficiency virus type 1 lipopeptide vaccine. J Virol $2000 ; 74: 1694-703$.

25. Gahery-Segard H, Pialoux G, Figueiredo S, et al. Long-term specific immune responses induced in humans by a human immunodeficiency virus type 1 lipopeptide vaccine : characterization of CD8 $8^{+}$-T-cell epitopes recognized. J Virol 2003; 77 : 11220-31.

26. Gahéry H, Choppin J, Bourgault I, et al. HIV preventive vaccine research a the ANRS : the lipopeptide vaccine approach. Thérapie $2005 ; 60: 243-8$

27. Launay 0 , Durier C, Desaint $C$, et al. Cellular immune responses induced with dose-sparing intradermal administration of HIV vaccine to HIVuninfected volunteers : results of a phase 1 randomized trial. Plos One 2007 ; 2 : e725

28. Gahéry H, Daniel N, Charmeteau B, et al. New $C D 4^{+}$and $C D 8^{+} T$-cell responses induced in chronically HIV-1 infected patients after immunizations with an HIV-1 lipopeptide vaccine. AIDS Res Hum Retr $2006 ; 22: 684-94$

29. Lévy Y, Durier C, Lascaux AS, et al. Sustained control of viremia following therapeutic immunization in chronically HIV-1-infected individuals. AIDS $2006 ; 20: 405-13$.

30. Racape J, Connan F, Hoebeke J, et al. Influence of dominant HIV-1 epitopes on HLA-A3/peptide complex formation. Proc Natl Acad Sci USA $2006 ; 103: 18208-13$

\section{TIRÉS À PART}

J. Choppin
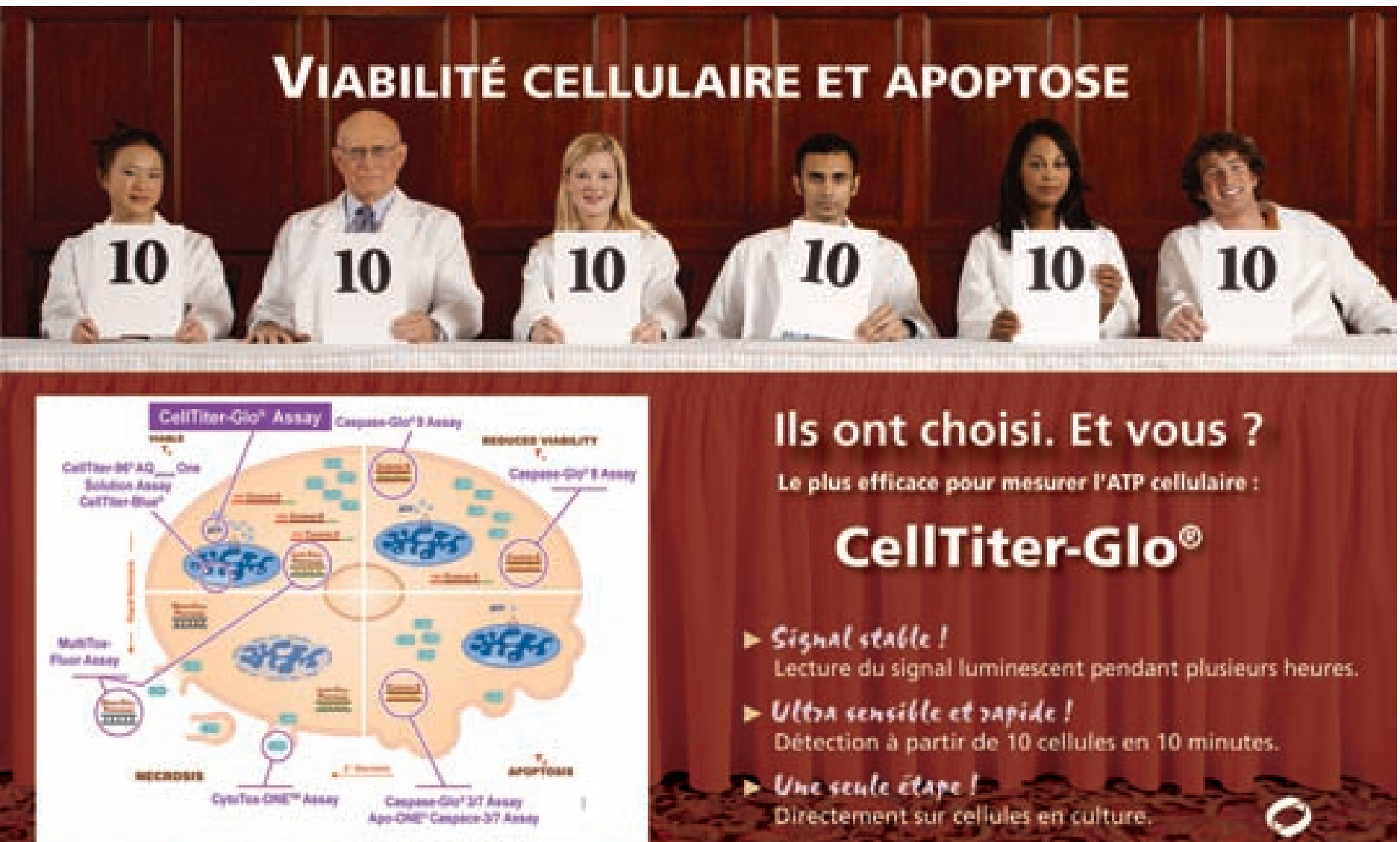

Ils ont choisi. Et vous?

Le plus efficace pour mesurer I'ATP cellulaire :

\section{CellTiter-Glo ${ }^{\oplus}$}

Signal stalle ! Lecture du signal luminescent pendant plusieurs heures.

- Ulba sensille et rapide! Détection à partir de 10 cellules en 10 minutes.

Une seule clape! Directement sur cellules en culture. 